\section{Difference threshold for intensity of tactile stimuli*}

\author{
JAMES C. CRAIG \\ Indiana University, Bloomington, Indiana 47401
}

The difference threshold (DL) for brief tactile stimuli (taps) and vibrotactile stimuli was determined using a 2IFC procedure. The measurements were made at several intensities both in quiet and in the presence of a background vibration. The results show that in the absence of background vibration the DLs for higher intensity stimuli are similar for both taps and vibration, whereas at lower intensities the DL is larger for taps. In the presence of background vibration the DL for vibratory stimuli is elevated to a much greater extent than it is for tap stimuli. The DL is affected by both the intensity of the signal and the intensity of the background vibration.

The difference threshold (DL) for intensity of vibratory stimuli on the skin has been examined several times. At moderate-to-high intensities of vibration, where the $D L$ is smallest, the estimate of $\Delta I / I$ ranges from approximately .30 (Sherrick, 1950) to .11 (Schiller, 1953) to .05 (Knudsen, 1928). It has been suggested that the apparent difference in the size of the threshold may be due to differences in techniques used by various investigators to measure the threshold, or possibly due to stimulus artifact (Geldard, 1962; Hawkes, 1961). Although the reconciliation of the apparently contradictory results might be of some interest, the present study is less concerned with the absolute value of the $D L$ than with relative changes in it as a function of several experimental manipulations.

There does seem to be a general agreement about several factors that affect the DL. First, a plot of $\Delta I / I$ as a function of I shows that at low intensities $\Delta I / I$ is large and gradually decreases as $I$ is increased. Second, changes in the frequency of vibration have little effect on the size of the DL (Knudsen, 1928; Schiller, 1953; Sherrick, 1950).

The present study was undertaken to examine the $\mathrm{DL}$ for intensity of sinusoidal stimuli (vibration) as compared to brief tactile stimuli (taps) both in quiet and in a background of extraneous vibration. Data that permit a comparison of DLs for vibration and taps have not been reported, although they would be useful in selecting highly discriminable stimuli in

* This study was supported by a Biomedical Sciences Support grant from PHS to Indiana University. Part of the research reported in this paper was conducted while the author held a summer faculty research fellowship from Indiana University. cutaneous communication systems (Geldard, 1960). Moreover, in such systems several loci on the body may be stimulated simultaneously, and the determination of the discriminability of intensity at a particular locus may require measurement of the $D L$ in the presence of background vibration.

A recent study (Raab \& Taub, 1969) of brief auditory stimuli suggests another reason a comparison of the DL for vibration and taps would DL for intensity of brief auditory stimuli (clicks) in quiet and in the presence of background noise. The results were somewhat surprising. First, the DLs were large compared to DLs for sinusoidal stimuli. In order to detect a change in intensity, Os required a difference in some cases of more than $3 \mathrm{~dB}$ between the two stimuli. Second, a plot of $\Delta I / I$ as a function of $I$ was bow-shaped, with the Weber fraction being larger in the middle range of intensities. This result is also unlike results obtained with longer auditory stimuli. Third, the addition of background noise reduced the size of the DL. Enough parallels between the auditory and the cutaneous systems have been drawn by various authors (Békésy, 1960) to suggest that a comparison of tactile and auditory stimuli would be interesting.

\section{METHOD}

\section{Observers}

The two Os were paid undergraduate females who received several hours of practice before data collection was begun.

\section{Apparatus}

For the sinusoidal stimuli, the output of a Hewlett-Packard 200AB oscillator was led to a Grason-Stadler 829C electronic switch that, in turn, drove a McIntosh 50-W amplifier, the be interesting. The study examined the output of which went to one of two sets of attenuators. One set of attenuators had a $110-\mathrm{dB}$ range in 1-dB steps. The second attenuator was identical to the first, with the addition of another attenuator, 1-dB range in .1-dB steps. A Gerbrands ratio programmer randomly determined whether the first stimulus generated would pass through the first attenuator or the second attenuator. A latching relay, energized on each trial after the first stimulus was presented, switched the output of the amplifier from the input of one attenuator to the input of the other attenuator, i.e., if the first signal generated went to the first attenuator, the second signal went to the second attenuator. The output of the attenuators was led to a matching transformer and then to a Goodmans V-47 vibrator. When taps were used as stimuli, a 2-msec pulse from a Tektronix 161 pulse generator was amplified, attenuated, and imposed on the vibrator. The programming of the stimuli was controlled by Tektronix waveform generators and pulse generators.

The background vibration was generated by a Grason-Stadler $455 \mathrm{C}$ noise generator, low pass filter setting at $20 \mathrm{kHz}$. The output of the noise generator was attenuated, mixed by means of a 500-ohm mixer with the signal, passed through a matching transformer, and led to the vibrator.

In order to control skin-contactor coupling factors (Craig \& Sherrick, 1969), the Goodmans V-47 vibrator was placed on the weighing pan of a balance. Sufficient weights were added to the balance to offset the weight of the vibrator. Twenty grams of weight were then added to insure proper contact of the vibrator with the O's finger. The piston of the vibrator protruded through a fixed surround $8 \mathrm{~mm}$ in diam. The vibrator was fitted with a circular contactor $6 \mathrm{~mm}$ in diam.

Procedure

Os were seated comfortably, with

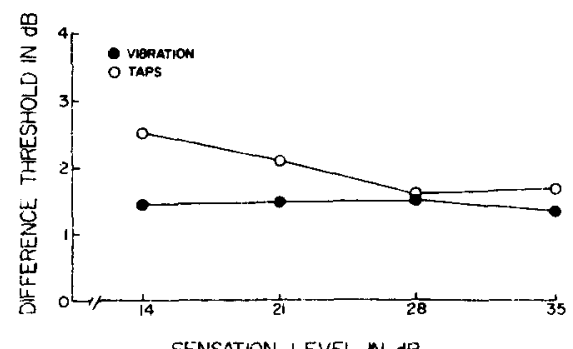

Fig. 1. Difference thresholds for vibrotactile stimuli and taps in the absence of background vibration. 


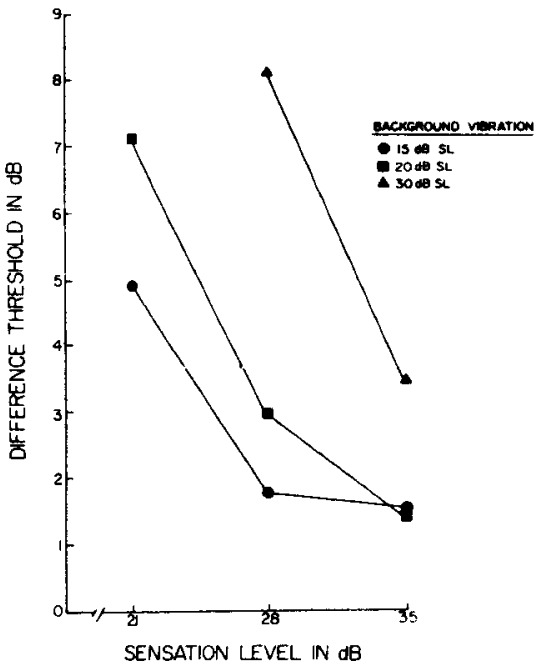

Fig. 2. Difference threshold for vibrotactile stimuli in the presence of a background vibration. The parameter is the intensity of the background vibration equated in sensory magnitude to the vibrotactile stimulus at 15,20 , and $30 \mathrm{~dB}$ SL.

their arms extended on a table. An O's right index finger was placed over the surround and the vibrator brought in contact with the finger. Sandbags were placed over the $O$ 's arm to hold it firmly in place. Os wore earphones through which white noise was fed to mask any sounds the equipment might make.

An absolute threshold was obtained by a modified method of limits for one of two stimuli, depending upon the experimental conditions: a 200 -msec burst of $160-\mathrm{Hz}$ vibration with a rise time of $10 \mathrm{msec}$, or a tap generated by a 2-msec square wave. The signal was raised to a 14-, 21-, 28-, or 35-dB sensation level (SL). A pilot study in which Os matched the intensity of a tap to the intensity of a monaurally presented click indicated that the range of subjective intensity of taps employed in the present study covered most of the range of subjective intensity of clicks in the Raab and Taub (1969) study. Each trial consisted of the $O$ pressing a button to start the trial, followed $1,000 \mathrm{msec}$ later by the first stimulus, followed by the second stimulus $1,000 \mathrm{msec}$ after the onset of the first stimulus. The $O$ was instructed that she would receive two signals. One of the two signals would feel more intense than the other. The $O$ was to indicate which one of the signals felt more intense by pressing the appropriate button with her left hand. Feedback was given by means of a light.

The procedure used to obtain the difference threshold consisted of a merger of a two-interval, temporal forced-choice technique with a block-up-and-down method (Campbell, 1963). Four trials constituted a block. When the $O$ made four correct responses out of four trials, the difference between the two signals was reduced .3 dB, i.e., the less intense of the two stimuli was increased in intensity by $.3 \mathrm{~dB}$. When the $O$ made three correct responses, the intensity of the stimuli was left unchanged. When the $O$ made two or fewer correct responses, the the intensity difference between the two stimuli was increased by .3 dB. Approximately 100 trials, or 25 blocks, were run to arrive at an estimate of the DL. The DL was estimated by calculating the median intensity difference between the two signals. The two types of stimuli, taps and vibration, were presented separately in a given sequence of $\mathbf{1 0 0}$ trials.

When background vibration was used, the $O$ was presented with two 500 -msec bursts of vibration produced by the output of the noise generator. Centered within each burst was the signal, either a burst of vibration or a tap that the $O$ was to discriminate. The time between the onsets of the stimuli to be discriminated was 1,000 msec. As before, the O's task was to indicate which interval contained the more intense stimulus. In order to set the intensity of the background vibration, the $O$ was presented with a burst of $160-\mathrm{Hz}$ vibration, $200 \mathrm{msec}$ in duration, followed by a 500-msec burst of the background vibration. The 200-msec stimulus was 15,20 , or $30 \mathrm{~dB}$ SL. The intensity of the background vibration was adjusted until the $O$ was satisfied that the two stimuli were equal in perceived magnitude. The same intensity levels of background vibration set by the $O$ for vibrotactile stimuli were used when taps were being discriminated.

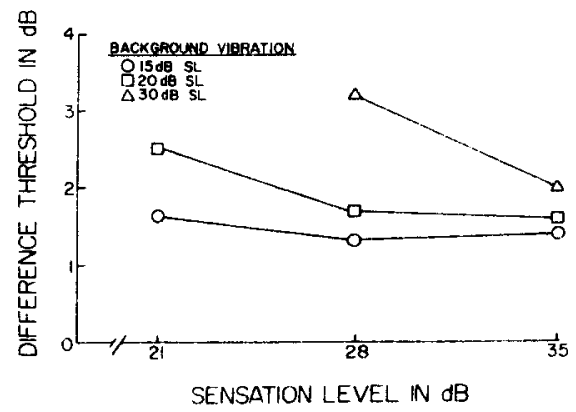

Fig. 3. Difference threshold for taps in the presence of a background vibration. The parameter of background vibration is the same as in Fig. 2 .

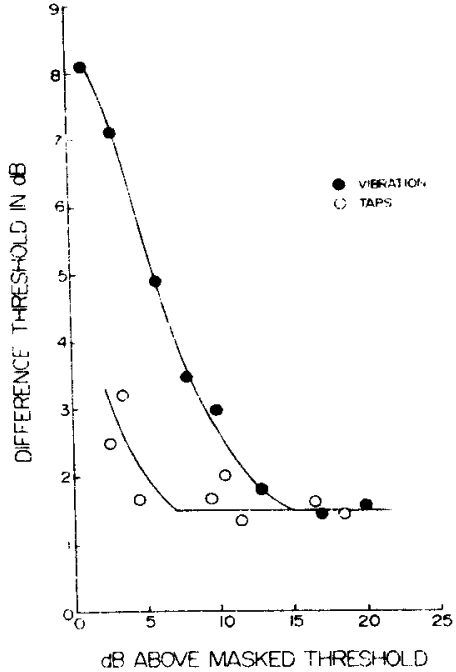

Fig. 4. Difference threshold for vibrotactile stimuli and taps as a function of the intensity level of the stimulus above masked threshold.

RESULTS AND DISCUSSION

The relative DL $[20 \log$ $((I-\Delta I) / I)]$ in decibels, plotted as a function of intensity (I) for both taps and bursts of vibration, is shown in Fig. 1. Each point represents the median of 10 measurements (approximately 100 trials per measurement). Looking first at the results for vibrotactile stimuli, the DL in decibels is approximately 1.5. The value, $1.5 \mathrm{~dB}$, represents a $16 \%$ change in amplitude, a result similar to those obtained by Spector (1954) using a different technique. Unlike other measurements of the DL, the threshold for vibrotactile stimuli in the present study does not increase as the intensity of the stimuli decreases. This is probably due to the fact that the lowest intensity, $14 \mathrm{~dB} \mathrm{SL}$, is high enough to be on the relatively flat portion of the functions generated in other studies. Spector's (1954) results show that the difference threshold rises sharply with decreasing intensity below $15 \mathrm{~dB}$ SL and remains relatively constant above 15 dB SL. Comparing the results from taps and bursts of vibration, the taps show a slightly higher DL, particularly at lower intensities.

The results for both taps and bursts of vibration in the presence of background vibration are shown in Figs. 2 and 3. Each point represents the median of 10 measurements. Comparing Fig. 1 with Figs. 2 and 3 shows that, in general, the background vibration increases the DL and that the increase in the $D L$ is much more pronounced for vibrotactile stimuli than for tap stimuli. It also appears that the DL is a joint function of the 
intensity of the background vibration and the intensity of the stimulus.

The question raised by Raab and Taub (1969) concerning the effect of noise on DLs for clicks does not receive an unequivocal answer. The presence of background vibration appeare to lower the threshold slightly at 21 dB SL, but no direct comparison is available for the 14-dB SL tap. Additional data vould be required to decide whether or not this is a genuine result. However, even if it were, such a result is much different from that reported for clicks (Raab \& Taub, 1969). First, the slope of the Weber function is not bow-shaped as it was for clicks. Second, the greater the background noise, the more the DL for clicks was reduced. Such a result was not observed for taps, but, in fact, somewhat the opposite case appears to occur. Third, the background noise had its greatest effect on the middle range of intensities of clicks, not on the lower intensities as appears to be the case for taps.

In order to compare more directly the DLs for taps and vibratory stimuli, measurements were made of the amount of masking produced by the three levels of background vibration. Absolute thresholds were obtained by a modified method of limits for the $160-\mathrm{Hz}$ vibratory stimulus or the tap. The $O$ was presented with a $500-\mathrm{msec}$ burst of the background vibration set at one of the three intensity levels used previously, i.e., judged to be equal in perceived magnitude to a 200 -msec burst of $160-\mathrm{Hz}$ vibration raised to 15,20 , or $30 \mathrm{~dB}$ SL. Centered within the burst of background vibration was either the tap or the $160-\mathrm{Hz}$ vibratory stimulus. With the exception that the step size was $1 \mathrm{~dB}$ rather than $.3 \mathrm{~dB}$, the same procedure used to measure DLs was used to determine the intensity level of the tap or the vibratory signal at which the $O$ was correctly detecting the signal $75 \%$ of the time. The difference in intensity levels required for detection in the absence of the background vibration and in the presence of the background vibration was taken as a measure of the amount of masking. The background vibration levels equivalent to the $160-\mathrm{Hz}$ stimulus at 15,20 , and $30 \mathrm{~dB}$ SL produced 15,18 , and $27 \mathrm{~dB}$, respectively, of masking of the vibratory stimulus. The amounts of masking of the tap were 16.5, 18.5, and $24.5 \mathrm{~dB}$, respectively. These figures are medians, each based on $\mathbf{4 0 0}$ trials. The amounts of masking were subtracted from each SL, and the data from Figs. 2 and 3 were replotted in Fig. 4. The results show again that the DIs for taps are much smaller than the DLs for vibratory stimuli, even, in this case, when measured at the same intensity level above the masked threshold. This result holds only for stimuli less than $14 \mathrm{~dB}$ above masked threshold. Above $14 \mathrm{~dB}$ the DLs for both types of stimuli are approximately the same. Also, for vibratory stimuli $14 \mathrm{~dB}$ or more above either masked or quiet threshold the DLs are similar, $1.5 \mathrm{~dB}$.

The large increase in the DL for vibratory stimuli as the intensity level above masked threshold decreases suggests that had the DL for stimuli of weaker intensities (below $14 \mathrm{~dB}$ SL) been examined in the absence of background vibration perhaps a similar increase in the DL would be seen. As mentioned before, results from Spector (1954) show a rise in the DL below approximately $15 \mathrm{~dB}$ SL. The similarity in the shapes of the two functions, one obtained from Spector's data and one obtained from the present data, suggest that it may be worthwhile to examine DLs in the absence of background vibration at weaker intensities. The present data indicate that, for vibratory stimuli, plotting DLs as a function of decibels above threshold, either masked or quiet threshold, yields a single function. Additional data are needed to determine whether or not such a function may be extended to stimuli at intensities lower than $14 \mathrm{~dB}$ above quiet threshold.

REFERENCES New York: McGraw-Hill, 1960.

CAMPBELL, R. A. Detection of a noise signal of varying duration. Journal of the Acoustical Society of America, 1963, 35, $1732-1737$.

CRAIG, J. C.. \& SHERRICK, C. E. The role of skin coupling in the determination of vibrotactile spatial summation. Perception \& Psychophysics, 1969, 6, 97-101.

GELDARD, F. A. Some neglected possibilities of communication. Science. $1960,131,1583-1588$.

GELDARD, F. A. Virginia cutaneous project, Psychology Lab., University of Virginia, Charlottesville, Final Report to ONR, Project NR-140-598, 1948-1962. Ann Arbor, Mich: University Microfilms, 1964, OP 16,352.

HAWKES, G. R. Cutaneous discrimination of electrical intensity. American Journal of Psychology, 1961, 74, 45-53.

KNUDSON, V. O. "Hearing" with the sense of touch. Journal of General Psychology, $1928,1,320-352$

RAAB. D. H.. \& TAUB, H. B Click-intensity discrimination with and without background masking noise. Journal of the Acoustical Society of America, 1969, 46, 965-968.

$S C H I L L E R$, H Ü b e $r$ i e amplitudenunterschiedsschwellen des Vibrationssinnes beim Menschen. Unpublished PhD dissertation. University of Eriangen, 1953. Cited by G. Hawkes, American Journal of Psychology, 1961, 74, 45-53.

SHERRICK, C. E. Measurement of the differential sensitivity of the human skin to mechanical vibration. Unpublished MA thesis, University of Virginia, 1950.

SPECTOR, P. Cutaneous communication system utilizing mechanical vibration. Unpublished doctoral dissertation. University of Virginia, 1954.

(Accepted for publication August 17, 1971.) 